\title{
Synthesis of High-Precision Hydraulic Control Systems of an Excavator
}

\author{
Konstantin Galitskov ${ }^{1}$, Stanislav Galitskov ${ }^{1}$, and Ivan Dudanov ${ }^{1, *}$ \\ ${ }^{1}$ Samara State Technical University, 194, Molodogvardeyskaya St., 443001, Samara, Russia
}

\begin{abstract}
Practical implementation of the program controlling the movement of the excavator's working tool (including the use of satellite navigation system) imposes stringent requirements on the dynamic and static accuracy of automatic control systems for the hydraulic excavator's operating mechanisms, in conditions of non-stationary parameters of these mechanisms as control objects. The authors describe the effective solution of this problem by implementing structural synthesis of each servo hydraulic drive as a multi-loop system with one measured coordinate (MLSOMC). The EO-4121 excavator with four hydraulic drives (rotary platform, boom, arm and ladle) was used as an example to demonstrate that the implementation of three circuits in the multi-loop system with one measured coordinate in each of these drives with typical PID-controllers allows to ensure the robustness of the ACS for known uncertainties, as well as the required rates of control quality in static and dynamic modes.
\end{abstract}

\section{Introduction}

The current level of development in constructional engineering imposes stricter requirements for the operation of an excavator. This implies, first, the need to increase the productivity and accuracy of excavation work (pit excavation, trench digging, etc.), where the excavator, while using modern control devices for positioning in space, primarily laser and satellite navigation systems [1,2], can operate in the mode of an industrial robot. The second task is the expansion of the universal machine characteristics due to the stability of its operation at low, the so-called "creeping" speeds [3], which is especially important when using an excavator for assembly and repair work.

An excavator is a manipulator with several elements of mobility. Most often, there are four of them - a rotary platform, a boom, an arm and a ladle. From point of view of operation, it is a multidimensional equipment with pronounced interchannel connections [46]. An excavator operator is constantly in the process of solving the complex optimization task of choosing the modes of coordinating the supporting systems while the excavator performs its technological operations [3]. Taking into account that, in practice, the operator is unable to control more than two movements of the machine's working element at the same time, the developers of the drives, when choosing the power of the hydraulic pump, impose a limitation permitting simultaneous supply of a working liquid with the final flow

* Corresponding author: iv_dudanov@mail.ru 
rate to only two actuating engines. When equipping the excavator drives with closed automatic control systems, the restrictions on the number of simultaneously controlled coordinates, the minimum speeds of the support systems and the positioning accuracy of the ladle are removed. As a result, the technological capabilities of the excavator are significantly expanded.

The article deals with the features of synthesis of high-precision excavator servo hydraulic drives for application-based use both in software control systems, for example, with the satellite navigation system [1,2] and in the mode when the desired trajectory and speed of the working element (including "creeping" speeds) is set but an excavator operator using the joystick $[7,8]$.

\section{Materials and methods}

The results of mathematic modeling of the actuators of the hydraulic excavator (exemplified by EO-4121 with drives for the rotary platform, boom, arm and handle $[2,6]$ ) as a multi-dimensional control object with $\bar{q}=\left[q_{1} q_{2} q_{3} q_{4}\right]^{T}$, vector as its output coordinates, the elements of which are the angular coordinates for the position of the rotary platform $\left(q_{1}\right)$, boom $\left(q_{2}\right)$, arm $\left(q_{3}\right)$ and ladle $\left(q_{4}\right)$, and the controlling activity vector $\bar{U}=\left[U_{1} U_{2} U_{3} U_{4}\right]^{T}$ includes the input actions $U_{1} \div U_{4}$ for the actuators (e.g. electrohydraulic amplifier or solenoid valve) of the corresponding hydraulic drive system, show [3, 4], that the dynamics of the object under reasonable assumptions and known limitations [3] can be presented by a linearized model and described by a matrix equation:

$$
\bar{q}(p)=[H(p)+M(p)] \bar{U}(p)
$$

where $H(p), M(p)$ are the diagonal transfer matrix describing the object's own channels, and the transfer connection matrix:

$$
H(p)=\left[\begin{array}{cccc}
W_{11}(p) & 0 & 0 & 0 \\
0 & W_{22}(p) & 0 & 0 \\
0 & 0 & W_{33}(p) & 0 \\
0 & 0 & 0 & W_{44}(p)
\end{array}\right], \quad M(p)=\left[\begin{array}{cccc}
0 & W_{12}(p) & 0 & 0 \\
W_{21}(p) & 0 & W_{23}(p) & 0 \\
0 & W_{32}(p) & 0 & W_{34}(p) \\
0 & 0 & W_{43}(p) & 0
\end{array}\right]
$$

A distinctive feature of the object is that the parameters of its own transfer functions are:

$$
W_{i i}(p)=\frac{k_{i}}{A_{i}(p)\left(T_{3} p+1\right) p},
$$

where $A_{i}(p)=T_{2 i}^{2} p^{2}+T_{1 i} p+1, \quad i \in 1, \ldots 4$; are essentially non-stationary (Table 1 ), which is caused by a change in the ladle position and the kinematic elements of the executive systems in the working space of the excavator during technological operations.

The problem of synthesis of high-precision systems for automatic control of the excavator hydraulic drives can be solved by providing these drives with maximum achievable speed and low disturb sensitivity. To achieve the set goals, we consider it appropriate to design each separate ACS structure (Figure 1) as a multi-loop system with one measured coordinate (MLSOMC) [9] using standard PID-controllers in each closed loop [10]. To evaluate the robustness of the automatic control system, we use the linear Tsypkin-Polyak criterion [11]. 


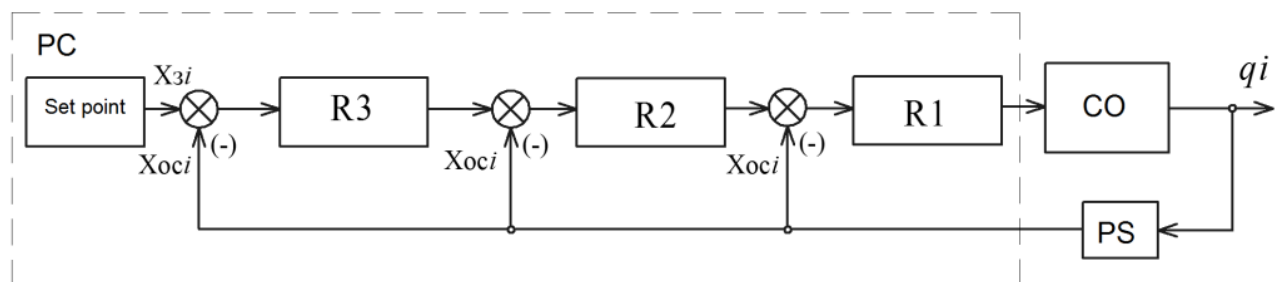

Fig. 1. Structural diagram of the automatic control system of an excavator hydraulic drives. PC programmable controller; R1, R2, R3 - ACS regulators; CO - control object; PS - position sensor for the excavator operating mechanism

Table 1. Parameters of mathematical models of the control object

\begin{tabular}{|c|c|c|}
\hline & & Linearized model of the control object \\
\hline $\begin{array}{l}\text { Degree of } \\
\text { freedom }\end{array}$ & $\begin{array}{l}\text { Variation of values for the } \\
\text { reduced moment of inertia or } \\
\text { mass }\end{array}$ & $\begin{aligned} W_{C O}(p)= & \frac{\Delta q(p)}{\Delta U_{\text {control }}(p)}=\frac{k_{C O}}{A(p)\left(T_{3} \cdot p+1\right) p} \\
& A(p)=T_{2}^{2} \cdot p^{2}+T_{1} \cdot p+1\end{aligned}$ \\
\hline $\begin{array}{l}\text { 1. Rotary } \\
\text { platform }\end{array}$ & $J_{n n}=(1,26 \div 2,96) \times 10^{5} \mathrm{~kg} \cdot \mathrm{m}^{2}$ & $\begin{array}{c}k_{C O}=63,6 \times 10^{-2} \mathrm{rad} / \mathrm{V} \cdot \mathrm{s} \\
T_{2}=(4,12 \div 8) \times 10^{-2} s ; \quad T_{1}=(18 \div 9,6) \times 10^{-2}{ }_{s}\end{array}$ \\
\hline 2. Boom & $m_{n p 2}=(9 \div 90) \times 10^{4} \mathrm{~kg}$ & $\begin{array}{c}k_{C O}=(3,4 \div 13,6) \times 10^{-2} \mathrm{rad} / \mathrm{V} \cdot \mathrm{s} \\
T_{2}=(14 \div 4,0) \times 10^{-2} s ; \quad T_{1}=(30 \div 4,8) \times 10^{-2} \mathrm{~s}\end{array}$ \\
\hline 3. Arm & $m_{n p 3}=(1 \div 10) \times 10^{4} \mathrm{~kg}$ & $\begin{array}{c}k_{c O}=(1,11 \div 3,4) \times 10^{-2} \mathrm{rad} / \mathrm{V} \cdot \mathrm{s} \\
T_{2}=(7 \div 0,32) \times 10^{-2} s ; \quad T_{1}=(11,2 \div 11) \times 10^{-2} \mathrm{~s}\end{array}$ \\
\hline 4. Ladle & $m_{n p 4}=(5 \div 50) \times 10^{3} \mathrm{~kg}$ & $\begin{array}{c}k_{C O}=(0,8 \div 2,4) \times 10^{-2} \mathrm{rad} / \mathrm{V} \cdot \mathrm{s} \\
T_{2}=(4 \div 0,31) \times 10^{-2}{ }_{s} ; \quad T_{1}=(6,4 \div 11,1) \times 10^{-2}{ }_{s}\end{array}$ \\
\hline
\end{tabular}

\section{Results}

When synthesizing the MLSOMC regulators (Figure 1) and its parametric optimization, we use the procedures proposed in [3,9] on the synthesis of high-speed drives invariant to load disturbances and supplement it with an estimate of the system's robustness [11].

Due to non-stationary nature of the parameters of the control object, the characteristic polynomial $\mathscr{t}_{1 k}(p)$ of the first closed loop is determined by the interval polynomial [11]

$$
\mathscr{\mathscr { A }}_{k}(p)=\left\{A_{1 k}(p)=a_{0}+a_{1} p+a_{2} p^{2}+a_{3} p^{3}+a_{4} p^{4}:\left|a_{i}-a_{i}^{0}\right| \leq \gamma \cdot \alpha_{i}, i=0,1, \ldots, 4\right\},
$$

here $a_{i}^{0}$ are the coefficients of the nominal polynomial

$$
A_{1 k .0}(p)=a_{0}^{0}+a_{1}^{0} p+a_{2}^{0} p^{2}+a_{3}^{0} p^{3}+a_{4}^{0} p^{4}, \quad a_{i}^{0}=\frac{\underline{a}_{i}+\bar{a}_{i}}{2}
$$

$\gamma$ - the range of uncertainty, $0 \leq \gamma \leq 1$. We believe that the system has a maximum indeterminateness $\gamma=1$, then $\alpha_{i}$ scale variation of the coefficient $a_{i}$ is:

$$
\alpha_{i}=\frac{\bar{a}_{i}-\underline{a}_{i}}{2}
$$


In the circuit under consideration, the boundary values of the coefficients are:

$$
\begin{aligned}
& \overline{a_{4}}=\frac{\overline{T_{3}} \overline{T_{2}^{2}}}{K_{p 1}}, \underline{a_{4}}=\frac{\overline{T_{3}}}{\overline{K_{p 1}^{2}}}, \overline{a_{3}}=\frac{\overline{T_{2}^{2}}+\overline{T_{1}} \overline{T_{3}}}{\underline{K_{p 1}}}, \underline{a_{3}}=\frac{\underline{T_{2}^{2}}+\underline{T_{1}}}{\overline{K_{p 1}}}, \\
& \overline{a_{2}}=\frac{\overline{T_{1}}+\overline{T_{3}}}{\underline{K_{p 1}}}, \underline{a_{2}}=\frac{\underline{T}_{1}+T_{3}}{\overline{K_{p 1}}}, \overline{a_{1}}=\frac{1}{\overline{K_{p 1}}}, \underline{a_{1}}=\frac{1}{\underline{K_{p 1}}}, \overline{a_{0}}=\underline{a_{0}}=1,
\end{aligned}
$$

where $K_{p 1}$ is $Q$ of the circuit, and the scale of these factors:

$$
\alpha_{4}=\frac{\overline{a_{4}}-\underline{a_{4}}}{2}, \alpha_{3}=\frac{\overline{a_{3}}-\underline{a_{3}}}{2}, \alpha_{2}=\frac{\overline{a_{2}}-\underline{a_{2}}}{2}, \alpha_{1}=\frac{\overline{a_{1}}-\underline{a_{1}}}{2}, \alpha_{0}=0 .
$$

The stability radius of the interval family is determined from the condition [11]:

$$
\gamma_{\max }=\min \left\{\gamma_{n}^{*}, \gamma_{n 0}, \gamma_{n \infty}\right\}
$$

where $\gamma_{n 0}=\frac{a_{0}^{0}}{\alpha_{0}}, \quad \gamma_{n \infty}=\frac{a_{4}^{0}}{\alpha_{4}}, \gamma_{n}^{*}-$ the side of the square inscribed in the Tsypkin hodograph diagram $Z(\omega)$.

Taking into account the equations (5) and (6), we conclude that in the circuit described $\gamma_{n \infty} \rightarrow \infty$, so $\gamma_{n}^{*}$ is determined from the condition:

$$
\gamma_{n}^{*} \leq \gamma_{n \infty}
$$

Value $\gamma_{n \infty}$ for the first loops of four excavator drives (Table 2) were used for parametric optimization of regulators following the criterion of robustness (Table 3 ).

Table 2. Values $\gamma_{n \infty}$ for the first circuits of the servo hydraulic drives

\begin{tabular}{|c|c|c|c|c|}
\hline Degree of freedom & Rotary platform & Boom & Arm & Ladle \\
\hline $\begin{array}{c}\text { Interval family } \gamma_{n \infty} \\
\text { radius of stability }\end{array}$ & 1.722 & 1.042 & 1.001 & 1.004 \\
\hline
\end{tabular}

Analysis of characteristic polynomials of the second and third closed circuits of the synthesized multi-loop systems with one measured coordinate (Fig. 1) by their interval polynomials

$$
\begin{aligned}
& \mathscr{\sigma}_{2 k}(p)=\left\{B_{2 k}(p)=b_{0}+b_{1} p+b_{2} p^{2}+b_{3} p^{3}+b_{4} p^{4}+b_{5} p^{5}:\left|b_{i}-b_{i}^{0}\right| \leq \gamma \cdot \beta_{i}, i=0,1, \ldots, 5\right\}, \\
& \tau_{3 k}(p)=\left\{C_{3 k}(p)=c_{0}+c_{1} p+c_{2} p^{2}+c_{3} p^{3}+c_{4} p^{4}+c_{5} p^{5}:\left|c_{i}-c_{i}^{0}\right| \leq \gamma \cdot \mu_{i}, i=0,1, \ldots, 5\right\},
\end{aligned}
$$

allows us to conclude that, with respect to the investigated structural circuits of the servo drives, the stability radii of the interval families of the second and third circuits are equal to the stability radius of the first circuit. The parameters of the synthesized regulators in these circuits allow to ensure the robustness of the ACS (Table 3). It is characteristic that in the first circuits the hodograph curves $Z(\omega)$ were practically touching the stability radius, while in the second circuits these hodograph curves moved away from the radius $\gamma_{n \infty}$. To 
display the mutual arrangement of $Z(\omega)$ and a square with a side $\gamma_{\max }$, we needed to choose such a scale (Table 3 ), that the stability radius practically degenerated into a point. Thus, the selected version of the structural synthesis of servo hydraulic actuators in the form of MLSOMC allows to effectively ensuring the robustness of the drives in conditions of substantial nonstationarity of the control object.

The study of transient processes in synthesized automatic control systems (taking into account the interchannel $W_{i j}(i \neq j)$ connections in a non-stationary object), performed in relation to the boom drive under conditions when one, two or three circuits are used within the MLSOMC (Table 4), show that with the growing number of loops, the quality of control is less dependent on the non-stationarity of control objects. As a result, we get a high-precision robust system with a low speed variation.

Table 3. Tsypkin-Polyak hodographs for hydraulic control systems

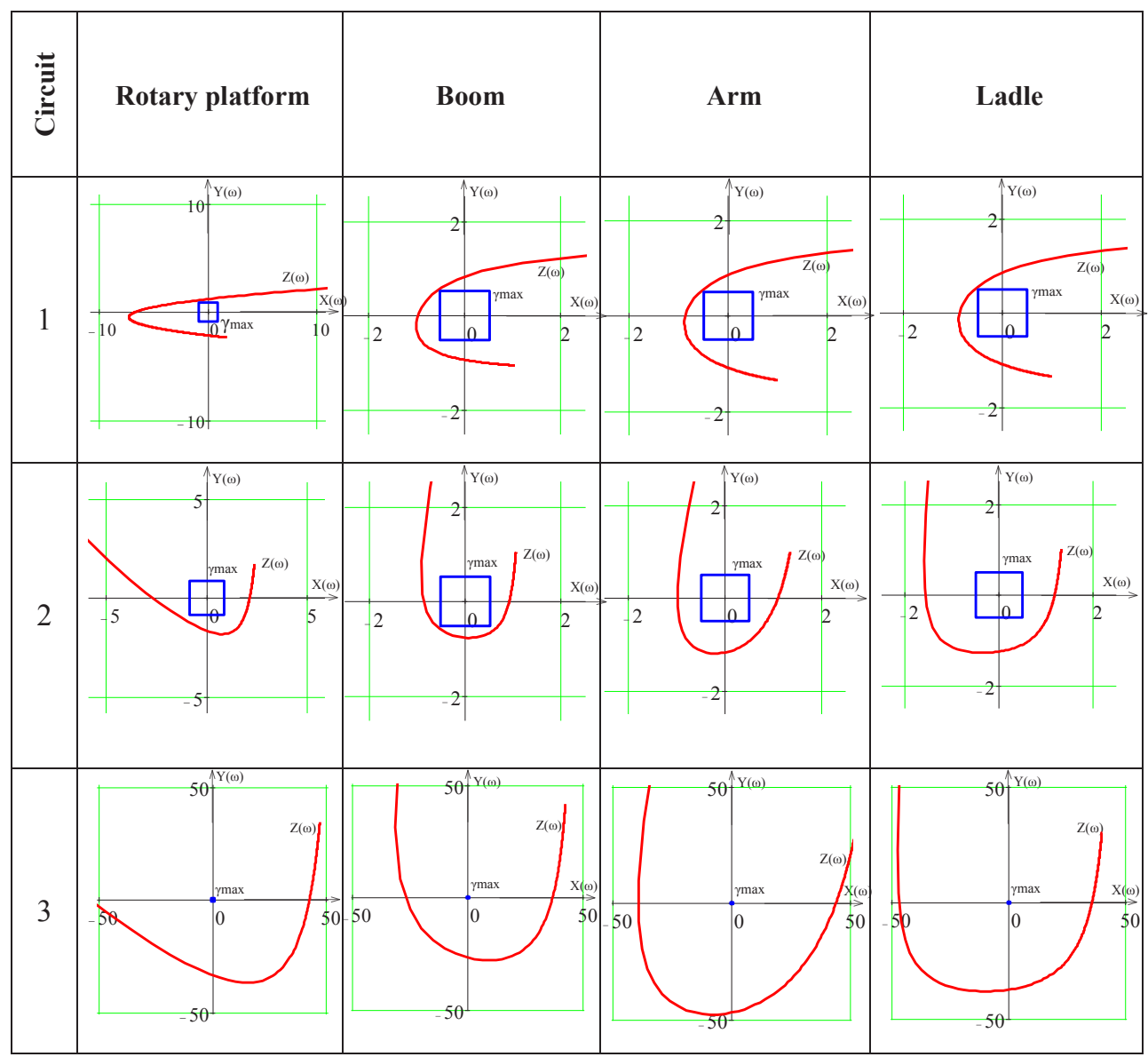

\section{Discussion}

The obtained results of the studies (exemplified by EO-4121 excavator) allow to reasonably assert that the selected variation of structural synthesis effectively ensures the ACS robustness of a hydraulic excavator for known range of uncertainty $y$ (Table 2) and the stability of the quality control parameters of the excavator working tool. 
Table 4. Comparing the quality of boom hydraulic drive control using one, two and three loops within the MLSOMC

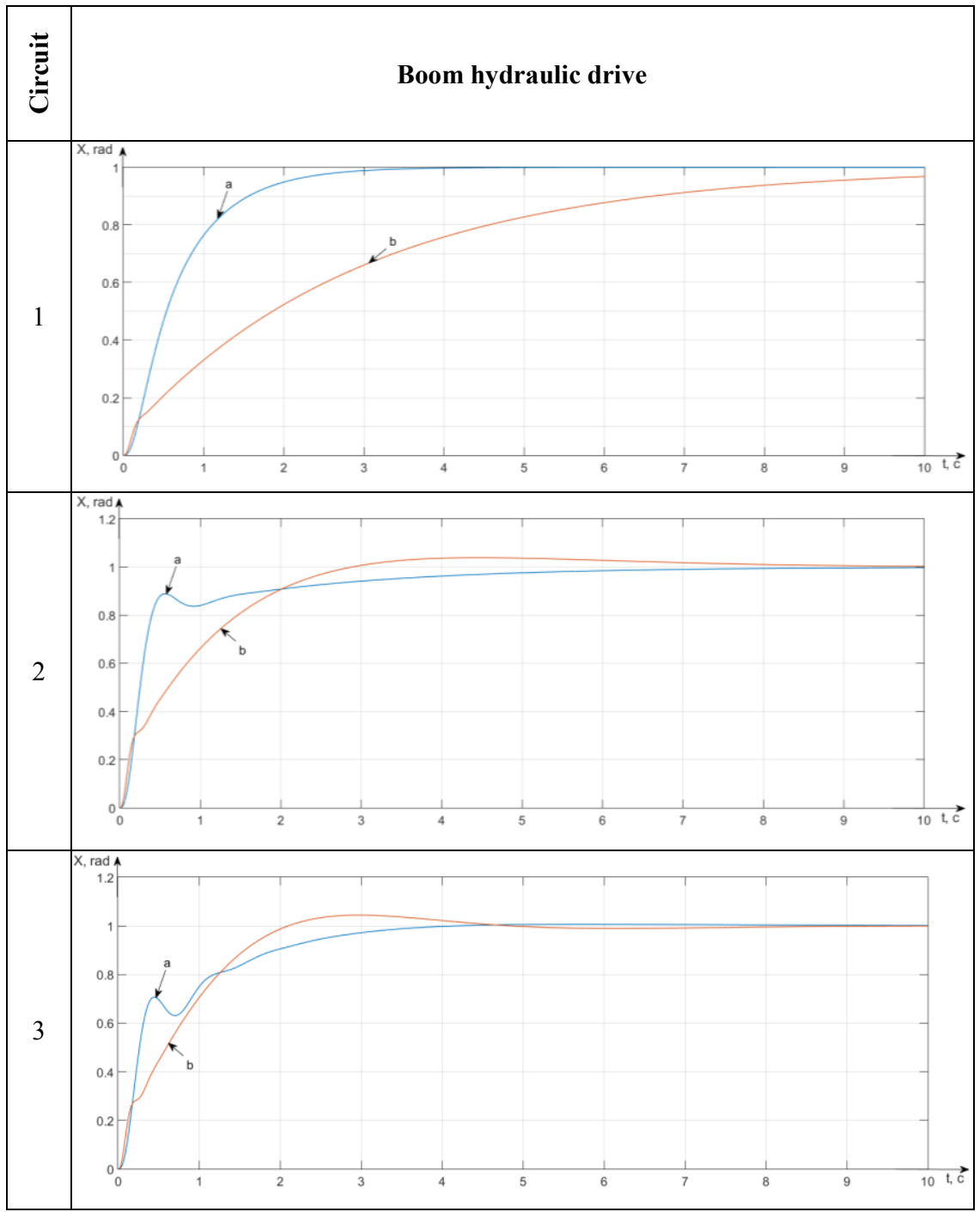

\section{Conclusions}

The authors demonstrate that the use of structural synthesis of separate channels of a multidimensional ACS for a hydraulic excavator in the form of MLSOMC allows to ensure the robustness of automatic control of hydraulic drives and the stability of their control quality indicators in conditions of extensive nonstationarity of the dynamic parameters of the control object.

The practical significance of the research involves the results obtained which can be used in the design and creation of high-precision robust systems for automatic control of hydraulic excavator drives centered around the idea of software control of this construction machine. 


\section{References}

1. I. Pavlov, Excavator Ladle Controlled via GPS/GLONASS, http://www.gruzovikpress.ru/article/4118-ekskavatorniy-kovsh-upravlyaetsya-cherezgps-glonass/

2. Control Systems for TOPCON 3DXI Excavators, https://maxiexkavator.ru/articles/excavators/ id=597

3. S.Ya Galitskov, Control Systems and Computer Simulation of the Hydraulic Drive of an Excavator (Samara, SSUACE, 2014)

4. S.Ya. Galitskov, I.V. Dudanov Galitskov, Mechanization of Construction 6, 2 (2008)

5. V.G. Ananin, Bulletin of Tomsk State University of Architecture and Construction 1, 9 (2013)

6. A.D. Terentyeva, Theory of Mechanisms 4(32), 9 (2016)

7. A.A. Akinfiev, Excavator Digging Control System, https://exkavator.ru/articles/designer/ $\mathrm{id}=5563$

8. D. A. Shekhovtsova, E.D. Komarov, Omsk Scientific Bulletin 3(133), 5 (2014)

9. S.Ya. Galitskov, K.S. Galitskov, Multi-Loop Control Systems with One Measured Coordinate (Samara, SSUACE, 2004)

10. S.Ya. Galitskov, I.V. Dudanov, The method of controlling the movement of the operating mechanism of a construction machine with a hydraulic drive and the device for its actuation. Patent 2340732 Russian Federation, IPC E02F 9/22 (2006)

11. B.T. Polyak, P.S. Shcherbakov, Robust Stability and Control (Moscow, Nauka, 2002) 